\title{
Shapes and Textures of Ferromagnetic Liquid Droplets
}

\author{
Shubho Banerjee* and M. Widom \\ Department of Physics, Carnegie Mellon University, Pittsburgh, Pennsylvania 15213
}

Received on 21 May, 2001

\begin{abstract}
Theoretical calculations, computer simulations and experiments indicate the possible existence of a ferromagnetic liquid state. Should such a state exist, demagnetization effects would force a nontrivial magnetization texture governed by the shape of the liquid droplet. Since liquid droplets are deformable, the droplet shape couples to the magnetization texture. This paper solves the joint shape/texture problem subject to the assumption of cylindrical droplet symmetry. The shape undergoes a change in topology from spherical to toroidal as exchange energy grows or surface tension decreases.
\end{abstract}

\section{Introduction}

In a spontaneously magnetized liquid state, long range magnetic order would exist in the liquid without application of any external field. The existence of a ferromagnetic liquid state in dipolar fluids has been indicated by mean field calculations [1-6] and computer simulations [7-10]. Experiments to observe liquid ferromagnetism in ferrofluids [11] are challenging because the fluids often freeze $[12,13]$ or phase separate [14] well above the predicted low temperatures for the onset of spontaneous magnetization. Recent experiments on strongly interacting $\mathrm{Fe}_{3} \mathrm{~N}$ ferrofluids [15] do show a hint of a possible ferromagnetic transition. Similarly, experiments on super-cooled Co-Pd alloys [16] indicate the possibility of ferromagnetism in supercooled liquid metals. In this case it is the strong exchange interaction, and not the dipole interaction, that would cause the spontaneous magnetization. The experimental evidence in both ferrofluid and supercooled Co-Pd is inconclusive, since the bulk of evidence addresses the temperature dependence of paramagnetic susceptibility above the Curie temperature [15-18]. Definitive proof of ferromagnetism below the Curie temperature remains elusive.

Although the existence of a ferromagnetic liquid state is yet to be proven experimentally, spontaneous polarization coupled with other order parameters has already been observed. Some electrically polarized liquid crystals [19] show a helical ordering of the dipole moments in the liquid. In superfluid ${ }^{3} \mathrm{He}$ the magnetic moment couples to the superconducting order parame- ter [20]. Many superfluid ${ }^{3} \mathrm{He}$ phases are therefore also magnetically ordered.

It is interesting to consider the magnetization texture (spatial variation of the orientation of magnetization) inside a droplet of such a ferromagnetic liquid [21]. The magnetization texture likes to avoid poles [22] to minimize its energy. However, this leads to defects inside the texture. For example, the rotating magnetization texture with cylindrical symmetry inside a sphere

$$
\mathbf{M}(r, \theta, \phi)=M \hat{\phi},
$$

where $\hat{\phi}$ is the unit vector for the variable $\phi$, avoids all poles but has a vortex line running through the center. Near the vortex of such a texture the magnetization is topologically unstable [23] and might escape into the third dimension [24] with a nonzero component along the vortex line. Whether this happens depends on the balance between demagnetizing and vortex energies. Simulated annealing of the magnetization inside a cubic box suggests that replacing vortices with point defects may be favorable [6]. However, for sufficiently large droplets the demagnetizing energy will dominate and only textures with vanishing demagnetizing energy (per unit volume) will occur.

Any defect is likely to have a system-shapedependent energy cost causing a deformable liquid droplet to deviate from a spherical shape. The complete calculation of the shape of an unconfined ferromagnetic liquid droplet in three dimensions, coupled with the calculation of its magnetization texture, remains an interesting and challenging unsolved problem.

This problem has a simple solution in two dimen-

\footnotetext{
${ }^{*}$ current address: Inst. Phys. Sci. Tech., U. of Maryland, College Park, MD 20742; email: shubho@ipst.umd.edu
} 
sions in zero field. The magnetization texture inside any soft (zero anisotropy) ferromagnetic solid thin film is given by van den Berg's algorithm [25] which avoids all poles, and thus all magnetostatic energy, at the expense of a domain wall through the film. A liquid droplet, which can change its shape, prefers a circular shape to minimize its surface energy. The magnetization lines inside a circle form concentric circles according to van den Berg's algorithm. For a circular shape the domain wall energy is also minimized because the domain wall shrinks to a point vortex. The circle thus solves the coupled texture and shape problem in zero field.

In a previous paper [26] addressing the thin film limit, we described the evolution of magnetization texture and droplet shape under the application of an inplane magnetic field. The vortex stretches to become a domain wall, which displaces towards the edge of the droplet. If exchange energy is taken into account, the droplet exhibits reflection symmetry-breaking, immediately revealing its magnetized state.

Our present goal is to analyze the shape and texture of an unconfined ferromagnetic liquid droplet. The problem is highly nontrivial even in the absence of applied field. To simplify the study, we restrict our attention to shapes and textures with an axis of continuous rotational symmetry. We find the texture exhibits a planar character with a vortex line along the symmetry axis. When surface tension is high or magnetization weak, the droplet shape remains nearly spherical, with slight "bulging at the waist" and dimpling where the vortex line meets the surface (Fig. 1). As surface tension drops or magnetization grows, the dimpling increases, shortening the vortex line. Eventually the droplet undergoes a change in topology to a toroidal shape (Fig. 2).

\section{Shape, texture and energy}

We consider droplets of volume $V$ and shape $\Omega$ with an internal magnetization texture $\mathbf{M}(\mathbf{r})$. Four terms contribute to the energy,

$$
E_{\text {tot }}=E_{\text {surf }}+E_{\text {exch }}+E_{\text {core }}+E_{\text {demag }} .
$$

The first term is the energy of the droplet surface, $\partial \Omega$,

$$
E_{\text {surf }}=\sigma \int_{\partial \Omega} d^{2} \mathbf{r}=\sigma A
$$

with $A$ the total droplet surface area and $\sigma$ the surface tension which we take to be isotropic. The surface energy is minimized for a spherical droplet.

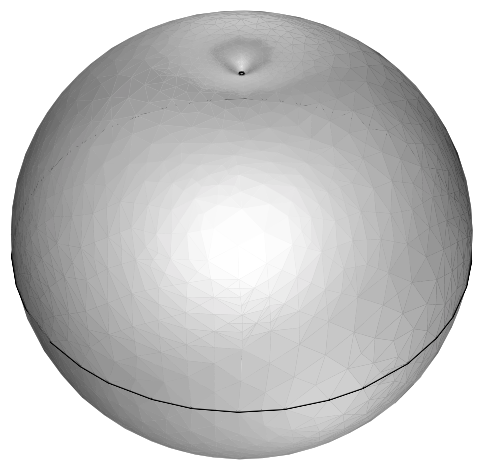

Figure 1. Apple shape obtained by minimizing eq. (13) for $\mathcal{M}^{2}=0.17$.

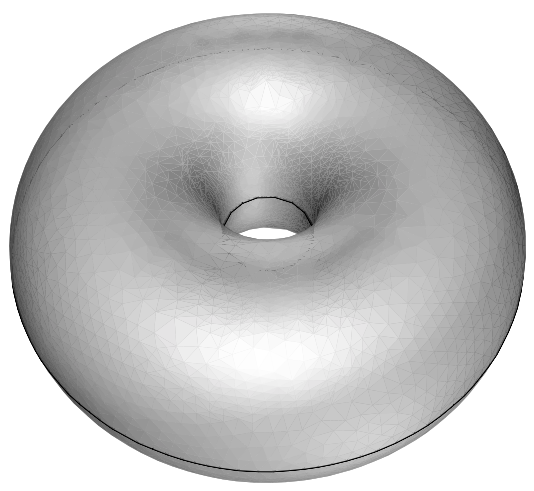

Figure 2. Donut shape obtained by minimizing eq. (13) for $\mathcal{M}=7.0$.

The second term is the exchange energy, which, for an isotropic medium, can be written as the integral over the droplet volume of

$$
U_{e x c h}=\frac{1}{2} \alpha \frac{\partial M_{k}}{\partial x_{i}} \frac{\partial M_{k}}{\partial x_{i}}
$$

where $\alpha$ is the exchange constant and the summation convention is employed [27]. The exchange constant has units of length squared. For a metallic ferromagnet the exchange constant typically takes a value of 
order $\lambda a^{2}$, where $a$ is a characteristic atomic size and $\lambda \sim 10^{4}$ is the ratio of exchange field to magnetostatic field [28]. For a ferrofluid, we can derive an effective exchange constant reflecting the energy cost of placing finite size particles into a rotating magnetic texture. By calculating the correction to the mean field in a cavity of diameter $a$ caused by rotation of the magnetization, we find $\alpha=4 \pi a^{2} / 15$. Again, $\alpha$ is of order $\lambda a^{2}$, where now $\lambda=1$ because the energy cost is magnetostatic in origin.

The third term is the energy associated with unmagnetized regions within the fluid. We presume that throughout most of the droplet the magnetization $\mathbf{M}(\mathbf{r})$ has constant magnitude, $M$, that minimizes the free energy density $f(M)$. Inspecting eq. (4) we see that exchange energy density may diverge at the core of a vortex, where $U_{\text {exch }} \sim \alpha / r^{2}$. Within a distance $r_{c}$ of the center of the vortex the exchange energy density $U_{\text {exch }}$ matches the free energy density cost $U_{\text {core }} \sim$ $f(0)-f(M)$. The core radius so-obtained does not depend on droplet shape or magnetization texture.

The final term in (2) is the demagnetizing energy that arises as a consequence of the long range $1 / r^{3}$ character of the dipole interaction. This weak fall-off of the interaction makes the total energy of the system dependent on global magnetization texture and system shape in general, challenging our notions of thermodynamic limits [29]. The shape dependent demagnetizing energy for dipolar systems can be best understood in terms of the demagnetizing field

$$
\mathbf{H}_{D}(\mathbf{r})=\int_{S} d^{2} \mathbf{r}^{\prime}\left(\mathbf{M}\left(\mathbf{r}^{\prime}\right) \cdot \hat{\mathbf{n}}\left(\mathbf{r}^{\prime}\right)\right) \frac{\mathbf{r}-\mathbf{r}^{\prime}}{\left|\mathbf{r}-\mathbf{r}^{\prime}\right|^{3}}-\int_{V} d^{3} \mathbf{r}^{\prime}\left(\nabla \cdot \mathbf{M}\left(\mathbf{r}^{\prime}\right)\right) \frac{\mathbf{r}-\mathbf{r}^{\prime}}{\left|\mathbf{r}-\mathbf{r}^{\prime}\right|^{3}}
$$

Here $\hat{\mathbf{n}}$ is the normal to the surface of the system. The first term on the right hand side reflects the surface poles that are created where the magnetization has a component normal to the surface. The second term is the contribution from the bulk charge density that is created by a nonzero divergence of the magnetization.

$\mathbf{H}_{D}$ is called the demagnetizing field because it inhibits the magnetization, as shown by the magnetostatic energy

$$
E_{D}=-\frac{1}{2} \int_{V} d^{3} \mathbf{r} \mathbf{H}_{D}(\mathbf{r}) \cdot \mathbf{M}(\mathbf{r})
$$

which can be rewritten

$$
E_{D}=\frac{1}{8 \pi} \int_{\text {all space }} d^{3} \mathbf{r}\left|\mathbf{H}_{D}(\mathbf{r})\right|^{2} .
$$

This energy is manifestly positive definite and hence inhibits the magnetization.

To avoid a demagnetizing energy by eliminating its demagnetizing field, magnetization textures in crystals break up into domains separated by domain walls. For suitable domain structure the demagnetizing energy can be removed entirely. The domain wall width is set by balancing the cost in exchange energy for rotating textures against the cost in magneto-crystalline anisotropy energy when a the magnetization does not point along an easy axis. Because a ferromagnetic liquid should lack magneto-crystalline anisotropy, the domain wall width diverges [21] unless it is limited by some other characteristic length [26].
The four terms in eq. (2) grow at differing rates as droplet volume increases while droplet shape and texture are held fixed. We find

$$
\begin{aligned}
E_{\text {surf }} & \sim \sigma R h \\
E_{\text {exch }} & \sim \alpha M^{2} h \log R / r_{c} \\
E_{\text {core }} & \sim U_{\text {core }} h \\
E_{\text {demag }} & \sim D M^{2} R^{2} h .
\end{aligned}
$$

In the above, we have taken magnetization texture (1) in a cylinder of height $h$ and radius $R$ for our calculation of $E_{\text {exch }}$, and we have taken $\mathbf{M}(\mathbf{r})$ constant inside an ellipsoid of demagnetization factor $D$ for our calculation of $E_{\text {demag }}$. For sufficiently large droplets, $E_{\text {demag }}$ dominates unless a texture is found to reduce it or remove it altogether. Anticipating that textures with vanishing $E_{\text {demag }}$ will emerge, the dominant energy becomes $E_{\text {surf }}$, so large droplets will favor compact shapes. For large $R$, we find $E_{\text {exch }}$ dominates $E_{\text {core }}$. At fixed $R$, the exchange energy mimics the core energy, with an energy cost proportional to the vortex length.

\section{Energy minimization}

We now confront the problem of simultaneously solving for the lowest energy magnetization texture within a droplet of a given shape while varying the shape to 
achieve the lowest total energy. To simplify our work, we choose to work within a limited subset of the family of possible textures and shapes. We impose cylindrical symmetry, motivated both by our suspicion that the true energy minimum may exhibit such a symmetry and by the considerable calculational simplifications that it allows.

Hence we assume the shape $\Omega$ and the magnetization texture $\mathbf{M}(\mathbf{r})$ are symmetric under rotations about the $\hat{z}$ axis. The magnetization vector field obeys

$$
\mathbf{M}(r, \phi, z)=\left(\begin{array}{ccc}
\cos \phi & -\sin \phi & 0 \\
\sin \phi & \cos \phi & 0 \\
0 & 0 & 1
\end{array}\right) \mathbf{M}(r, 0, z)
$$

The droplet shape $\Omega$ is defined by its boundary $\partial \Omega$ which we parameterize by the function $\pm z(r)$. This family of shapes exhibits reflection symmetry through the $z=0$ plane in addition to rotational symmetry about the $\hat{z}$ axis.

For large droplets the demagnetizing energy is dominant, as we showed in eq. (9). Consider the problem of minimizing $E_{D}$ within a symmetric shape. Since $E_{D} \geq 0$, we can solve this minimization with any texture that achieves $E_{D}=0$, which, by equation (7) requires $\mathbf{H}_{D}=0$ through all space. Inspecting (5), we can achieve $\mathbf{H}_{D}=0$ by eliminating all surface poles and volume divergence.

The simplest texture with $\mathbf{H}_{D}=0$ is given in eq. (1). This texture is purely planar, satisfies $\mathbf{H}_{D}=0$, but exhibits a vortex line running along the $z$-axis. The exchange energy density varies as

$$
U_{E X}=\frac{\alpha M^{2}}{2 r^{2}}
$$

and diverges at $r=0$. Therefore, we impose a short length cut-off of $r_{0}$. Inside the vortex $\left(r<r_{0}\right)$ we assume a uniform energy density $U_{\text {core }}$. The vortex radius $r_{0}$ can be chosen by balancing $\alpha M^{2} / r_{0}^{2}$ against $U_{\text {core }}$ at $r_{0}$ yielding $r_{0} \sim\left(\alpha M^{2} / U_{\text {core }}\right)^{1 / 2}$. Multiplying the core energy density by the cross-sectional area yields a vortex energy cost per unit length, $U_{V}=\pi r_{0}^{2} U_{\text {core }}$.

This texture is probably not the absolute lowest in energy. We expect that the magnetization will rotate out-of-plane close to the vortex core. Such a distortion comes with a small cost in demagnetizing energy, but brings a large savings in exchange energy. Since this distortion is limited to the region close to the vortex [24], we may consider it as part of the core structure, and it is not relevant for the bulk droplet shape and energy. Large scale out-of-plane rotations cause large demagnetizing energy costs unless they are spatially nonuniform. Spatial nonuniformity will raise exchange energy costs. Hence, we expect the texture (1) is optimal sufficiently far from the vortex.

The exchange energy can be reduced, and the vortex core energy eliminated altogether, by a change in topology from spherical to toroidal. Consider a torus with cylindrical symmetry about the $\hat{z}$ axis, with crosssection $\pm z(r)$ for $R_{i} \leq r \leq R_{o}$. Assuming texture (1), and noting $E_{\text {core }}=E_{\text {demag }}=0$, the total energy is the sum of surface and exchange energy

$$
E_{t o t}=2 \sigma \int_{0}^{2 \pi} d \phi \int_{R_{i}}^{R_{o}} r d r \sqrt{1+(d z / d r)^{2}}+\frac{\alpha M^{2}}{2} \int_{0}^{2 \pi} d \phi \int_{R_{i}}^{R_{o}} r d r \int_{-z(r)}^{z(r)} \frac{d z}{r^{2}} .
$$

The curvature energy competes with the surface energy to determine the shape of the droplet in this model. For shapes with spherical topology, we must add an additional term to eq. (11) of the form $U_{V} L_{V}$, where $L_{V}$ is the length of the vortex. However, since the exchange energy mimics the effect of the vortex core energy, we set $U_{V}=0$ in the following.

The relative strength of each term is governed by a single dimensionless magnetization parameter defined so that

$$
\mathcal{M}^{2} \equiv \frac{\alpha M^{2}}{\sigma L}
$$

where $L$ is a measure of the linear droplet dimension $\left(L \sim(\text { volume })^{1 / 3}\right)$. Scaling lengths by $L$ and energy by $4 \pi L^{2} \sigma$, we introduce a dimensionless form of the total energy

$$
\mathcal{E}_{t o t}[z(r)]=\int_{\mathcal{R}_{i}}^{\mathcal{R}_{o}} r d r \sqrt{1+(d z / d r)^{2}}+\frac{\mathcal{M}^{2}}{2} \int_{\mathcal{R}_{i}}^{\mathcal{R}_{o}} r d r \frac{z}{r^{2}}
$$


where script quantities are dimensionless.

To calculate the shape of the droplet we used the program Surface Evolver by Kenneth Brakke [30]. The program approximates a surface using a triangular mesh, and the vertices of the mesh evolve to minimize the total energy of the surface subject to various constraints of the problem (fixed volume in our case).

We find that for small values of $\mathcal{M}$ the droplet takes a dimpled and slightly oblate "apple" shape (see Fig. 1). This occurs because the droplet reduces its curvature energy by reducing the length of the vortex (hence the dimpling where the vortex meets the surface) and by placing the bulk of the fluid as far as possible from vortex (hence the oblate shape). As $\mathcal{M}$ increases, the dimpling grows and the vortex shortens. This occurs even in the absence of a vortex core energy and is driven by the diverging exchange energy density near the vortex. For sufficiently large $\mathcal{M}$, the vortex shrinks to zero length and the droplet changes topology, acquiring a "donut" shape (see Fig. 2).

For large $\mathcal{M}$ the hole at the center of the donut is large. As $\mathcal{M}$ decreases, the hole shrinks, and the walls of the hole become nearly vertical forming a cylinder of radius $R_{i}$. We can estimate this radius by balancing the $R_{i}$ dependence of the cylinder surface energy against the $R_{i}$ dependence of the magnetic energy outside the cylinder (and thus inside the torus). We estimate

$$
\frac{d E_{\text {surf }}}{d R_{i}} \approx 2 \pi L \sigma
$$

and

$$
\frac{d E_{e x c h}}{d R_{i}} \approx-2 \pi R_{i} L \frac{\alpha M^{2}}{2 R_{i}^{2}}
$$

from which it follows that $R_{i} \approx \alpha M^{2} / 2 \sigma$ (in dimensionless variables, $\left.\mathcal{R}_{i} \approx \frac{1}{2} \mathcal{M}^{2}\right)$. Thus the donut hole remains for all values of $\mathcal{M}$, vanishing as $\mathcal{M} \rightarrow 0$. Intriguingly, the structure of the donut hole for small $\mathcal{M}$ resembles the dimple on the apple surface at the same value of $\mathcal{M}$. The dimple is a cylindrical hole of radius $R_{i}$, but the hole extends only part way through the apple core, with the vortex occupying the remainder.

The energy of the apple is lower than the energy of the donut for small $\mathcal{M}$ and remains lower until just before the apple core shrinks to zero. For larger values of $\mathcal{M}$, the apple shape does not exist and the donut is the stable shape. For a droplet with volume such that $\mathcal{L}=12$ and vortex radius $r_{0}=0.1$, the pinch-off point is close to $\mathcal{M}=0.2$.

\section{Conclusion}

In this paper, we fix a simple magnetization texture and then calculate the shape. Surface evolver is suited to optimization of shape. To express the total energy of the droplet as a function of its shape, we required that the magnetization be cylindrically symmetric and confined to a plane. The vortex core was assumed to be straight, and we neglected the energy of the vortex core.

For a more rigorous study, a simultaneous calculation of the magnetization texture and the shape is needed. Since the magnetization may rotate out of plane near the vortex [23], and possibly break the cylindrical symmetry, the analysis will require breaking up the volume of the droplet into finite elements [31] and evolving the shape to minimize the sum of demagnetizing, curvature, vortex and surface energies. However, our simple analysis illustrates the nontrivial nature of the problem and gives an idea of shapes that might occur for ferromagnetic liquid droplets.

\section{Acknowledgements}

We acknowledge useful discussions and communications with R. B. Griffiths, A. A. Thiele and L. Berger. This work was supported in part by NSF grant DMR9732567 at Carnegie Mellon University and by NSF grant CHE-9981772 at University of Maryland.

\section{References}

[1] K. Sano and M. Doi, J. Phys. Soc. Jpn. 52, 2810 (1983).

[2] A. O. Tsebers, Magnetohydrodynamics 2, 42 (1982).

[3] H. Zhang and M. Widom, J. Magn. Magn. Mater. 122, 119 (1993); Phys. Rev. E 49, R3951 (1994).

[4] M. Widom and H. Zhang, Phys. Rev. Lett. 74, 2616 (1995).

[5] B. Groh and S. Dietrich, Phys. Rev. Lett. 722422 (1994); 74, 2617 (1995); Phys. Rev. E 50, 3814 (1994).

[6] B. Groh and S. Dietrich, Phys. Rev. E 57, 4535 (1998).

[7] D. Wei and G. N. Patey, Phys. Rev. Lett. 68, 2043 (1992); Phys. Rev. A 46, 7783 (1992).

[8] J. J. Weis, D. Levesque, and G. J. Zarragoicoechea, Phys. Rev. Lett. 69, 913 (1992).

[9] J. J. Weis and D. Levesque, Phys. Rev. E 48, 3728 (1993).

[10] M. J. Stevens and G. S. Grest, Phys. Rev. E 51, 5962 (1995); Phys. Rev. E 51, 5976 (1995).

[11] R. E. Rosensweig, Ferrohydrodynamics (Cambridge University Press, Cambridge, England, 1985).

[12] J. Zhang, C. Boyd, and W. Luo, Phys. Rev. Lett. 77, 390 (1996).

[13] D. Ederbeck and H. Ahlers, J. Magn. Magn. Mater. 192, 148 (1999).

[14] W. Luo, Nuovo Cimento D (Italy) 16, 1199 (1994). 
[15] H. Mamiya, I. Nakatani and T. Furubayashi, Phys. Rev. Lett. 84, 6106 (2000).

[16] J. Reske, D. M. Herlach, F. Keuser, K. Maier, and D. Platzek, Phys. Rev. Lett. 75, 737 (1995); T. Albrecht, C. Buhrer, M. Fahnle, K. Maier, D. Platzek, and J. Reske, Appl. Phys. A 65, 215 (1997).

[17] C. Buhrer, M. Beckmann, M. Fahnle, U. Grunewald and K. Maier, J. Magn. Magn. Mater. 212, 211 (2000).

[18] T. Schenk, D. Holand-Moritz, W. Bender and D. M. Herlach, J. Non-Cryst. Solids 250-252, 694 (1999); T. Schenk, D. Holand-Moritz and D. M. Herlach, Europhys Lett. 50, 402 (2000).

[19] R. B. Meyer, L. Liebert, L. Strzelecki, P. Keller, J. de Phys. (France) Lett. 30, 69 (1975).

[20] N. D. Mermin and D. M. Lee, Scientific American 235 56 (1976).

[21] P. G. de Gennes and P. A. Pincus, Solid State Comm. 7, 339 (1969)
[22] W. F. Brown, Magnetostatic Principles in Ferromagnetism (North-Holland, Amsterdam, 1962).

[23] N. D. Mermin, Rev. Mod. Phys. 51, 591 (1979).

[24] A. Hubert, J. de. Phys. (France) C8, 1859 (1988).

[25] H. A. M. van den Berg, J. Appl. Phys. 60, 1104 (1986).

[26] S. Banerjee and M. Widom, Phys. Rev. E 61, 4171 (2000).

[27] L. D. Landau and E. M. Lifshitz, "Electrodynamics of Continous Media" (Addison Wesley, 1960).

[28] C. Kittel, "Introduction to Solid State Physics" (6th edition) (Wiley, 1986) p. 423.

[29] S. Banerjee, R.B. Griffiths and M. Widom, J. Stat. Phys. 93, 109 (1998); To appear in J. Stat. Phys. (2001).

[30] See http://www.geom.umn.edu/software/evolver for details.

[31] O.C. Zienkiewicz, The Finite Element Method in Engineering Science 2nd ed. (McGraw-Hill, 1971). 